\title{
High VEGF Level is Produced by Human Umbilical Cord- Mesenchymal Stem Cells (hUC-MSCs) in Amino Acid-Rich Medium and Under Hypoxia Condition
}

\author{
Veronika Maria Sidharta ${ }^{1,2}$, Elizabeth Henny Herningtyas ${ }^{3, *}$, Christine Ayu Lagonda $^{4}$, \\ Dilafitria Fauza ${ }^{4}$, Yuyus Kusnadi ${ }^{4}$, Rina Susilowati ${ }^{5}$, Ginus Partadiredja ${ }^{6}$ \\ ${ }^{1}$ Doctoral Program, Faculty of Medicine, Public Health and Nursing, Universitas Gadjah Mada, Jl. Farmako Sekip Utara, Yogyakarta, Indonesia \\ ${ }^{2}$ Histology Department, School of Medicine and Health Science, Atma Jaya Catholic University of Indonesia, Jl. Pluit Raya No. 2, Jakarta, Indonesia \\ ${ }^{3}$ Clinical Pathology and Laboratory Medicine Department, Faculty of Medicine, Public Health and Nursing, Universitas Gadjah Mada, \\ Jl. Farmako Sekip Utara, Yogyakarta, Indonesia \\ ${ }^{4}$ Stem Cell and Cancer Institute, Jl. A. Yani No. 2, Jakarta, Indonesia \\ ${ }^{5}$ Histology and Biology Cell Department, Faculty of Medicine, Public Health and Nursing, Universitas Gadjah Mada, Jl. Farmako Sekip Utara, \\ Yogyakarta, Indonesia \\ ${ }^{6}$ Physiology Department, Faculty of Medicine, Public Health and Nursing, Universitas Gadjah Mada, Jl. Farmako, Sekip Utara, Yogyakarta, Indonesia \\ *Corresponding author. E-mail: ehennyh@ugm.ac.id
}

Received date: Apr 23, 2018; Revised: Jun 18, 2018; Accepted: Jun 19, 2018

\section{Abstract}

$\mathrm{B}$ ACKGROUND: Secretome production by stem cells depends on their culture conditions such as oxygen concentration and the composition of the culture media. In this study, we investigated the secretion of neurotrophic growth factors of human umbilical cord mesenchymal stem cells (hUC-MSCs) in amino acid-rich culture medium and under hypoxic condition.

METHODS: hUC-MSCs were cultured in normoxic and various hypoxic $(1 \%, 5 \%, 10 \%)$ conditions in an amino acid-rich culture medium. The end-point parameters (cell proliferation and survival, cell morphology and growth factor secretion) were measured at 3 time-points (48 hours, 72 hours and 96 hours). ELISA-based methods were used for neurotrophic factors detection, including neurotrophic growth factor (NGF), vascular endothelial factor (VEGF), and brain-derived neurotrophic factor (BDNF).

\section{Introduction}

Mesenchymal stem cells (MSCs) were first identified from bone marrow cells in 1966 by Friedenstein.(1) Recently, MSCs have been isolated from almost every tissue in the body, including human umbilical cord MSCs (hUC-
RESULTS: NGF secretion was not detectable at any time points both in normoxia and hypoxia. BDNF secretion under normoxia was induced at $48 \mathrm{~h}$ time point and reached the highest level at an average of $181.9 \pm 13.01 \mathrm{pg} / \mathrm{mL}$ at 96 hours, whereas hypoxia exposure to hUC-MSCs only induced the BDNF secretion at low level. VEGF secretion was barely detectable in normoxic condition. However, VEGF secretion reached the highest level at an average of $7707.55 \pm 2110.85 \mathrm{pg} / \mathrm{mL}$ in $5 \%$ hypoxia at 96 hours.

CONCLUSION: Combination of amino acid-rich culture medium and hypoxia condition dramatically induced high VEGF secretion by hUC-MSCs, especially at 5\% hypoxia, induced mild BDNF secretion and had no effect toward NGF secretion.

KEYWORDS: human umbilical cord mesenchymal stem cells, neurotrophic growth factor, amino acid-rich, hypoxia

Indones Biomed J. 2018; 10(3): 222-30
MSCs).(2,3) The International Society for Cellular Therapy (ISCT) defines that MSCs are characterized by the expression of the surface markers cluster of differentiation (CD)73, CD90 and CD105, and are negative for the expression of CD11b, CD14, CD19, CD79a, CD34, CD45 and human leukocyte antigen (HLA)-DR. Moreover, MSCs also have the ability to differentiate into osteoblasts, adipocytes, and 
chondroblasts in vitro.(4) Since this discovery, stem cell therapy has become a promising strategy to treat many degenerative problems, including neurodegenerative disorders.(5-7) Compared to MSCs from other sources, hUC-MSCs, especially from Wharton's jelly (WJ), have many advantages such as accesibility, painless procedures for donors, no ethical controversy, and lower risk of contamination.(8)

Three main functions of stem cell therapy are replacement of tissue via multipotent differentiation, immunomodulatory and anti-inflammatory effects, and secretion of molecules (secretome) that instigate or assist in tissue repair.(9)

However, some limitations are found in this strategy, including the cells' short lifespan, senescence-induced genetic instability, and possible malignant transformation of stem cells.(10) It is now commonly accepted that the potency of stem cell therapy is related mainly to the secretome, the complex set of molecules secreted by the stem cells or shed from their cell surfaces, that support cell survival and create a suitable environment for regeneration by endogenous cells. $(11,12)$

Secretome from hUC-MSCs contains factors related to neuroprotection, neurogenesis, and angiogenesis (13) which is beneficial in neuroregenerative therapy. The secretome's composition depends on stem cell's culture conditions. Finding the optimum method to enhance the secretome composition is believed to improve stem cells' therapeutic potential. Previous studies showed that secretome production are influenced by incubation duration within low oxygen tension, and culture media which stimulates secretome release.(11,14-16) Different composition of media will affect the growth rate, differentiation potential and surface marker expression of mesenchymal stem cells.(17) Lower glucose concentrations will lead to a decreased apoptosis, decreased senescence, and increased proliferation rate of MSC. $(15,16)$

Various compositions of culture media are available, which includes high glucose and high minerals culture medium, for instance, Dulbecco's Modified Eagle Medium: Nutrient Mixture (DMEM)-F12 or high glucose and moderate amino acid content provided in Minimum Essential Media (MEM). A comparison between different basal culture mediua supplemented with $10 \%$ fetal bovine serum (FBS) revealed that DMEM-KO (knockout) and DMEM-F12, compared with DMEM-LG (low glucose) and DMEM-HG (high glucose), are the most optimal culture medium for WJ-MSCs.(18) Both Minimum Essential
Medium Eagle-Alpha modification (MEM Alpha) and DMEM-F12 contain high amino acid, however MEM Alpha has much higher concentrations of glycine, alanine, asparagine, aspartic acid, cysteine hydrochloride, glutamic acid and proline. In addition, MEM Alpha has lower glucose concentration compared to DMEM-F12 (Supplemental Data). There is limited information about the ability of hUC-MSCs to release secretome in amino acid-rich culture medium under hypoxic conditions, in this case is MEM Alpha. Thus in this study, we investigated the effects of various hypoxic conditions on the secretion of neurotrophic growth factors in an amino acid-rich culture medium, MEM Alpha.

\section{Methods}

\section{Isolation and Culture of hUC-MSCs}

hUC-MSCs stock cells were obtained from Stem Cell and Cancer Institute (SCI), Jakarta, Indonesia. These cells were originally isolated from human umbilical cords which were aseptically collected during caesarian sections. The vessels were manually removed from umbilical cord segments and the tissue was dissected into $1 \mathrm{~mm} \times 1 \mathrm{~mm}$ pieces. The tissue pieces were planted in a culture dish for 14 days until mesenchymal stem cells were diffused out from the tissue and attached to the vessel. They were then passaged continuously from P0 until P6 using TrypLe (Gibco, NY, USA) when they attained $75-85 \%$ confluence. The cells were cultured in MEM Alpha (Gibco) that contained low glucose and rich non-essential amino acids, supplemented with 10\% FBS (Gibco), 1\% penicillin-streptomycin (Sigma, Missouri, USA) and incubated at $37^{\circ} \mathrm{C}$ with $5 \% \mathrm{CO}_{2}$ and 95\% humidity.

\section{Characterization of hUC-MSC by Immunophenotyping} Cell surface marker analysis for hUC-MSCs was conducted when the culture had reached $6^{\text {th }}$ passage to characterize and determine positivity percentage of hUC-MSCs in isolated samples, using monoclonal antibodies conjugated with phycoerythrin (PE) fluorescent dye. Flow cytometry analysis using BD FACSCalibur (BD Bioscience, New Jersey, USA) was performed to analyze these cell-surface epitopes; CD73, CD90, CD105 (as positive markers of MSCs), CD14, and CD45 (as negative markers) (R\&D Systems, Minnesota, USA). For each sample, a minimum of 10,000 events were acquired and result were analyzed with CellQuestPro software (BD Biosciences). 


\section{in vitro Hypoxia}

hUC-MSCs were cultured up to $6^{\text {th }}$ passage to be used for secretome production. Cells were seeded in T-25 flasks at a density of 7000 cells $/ \mathrm{cm}^{2}$ in the culture medium until reaching $80 \%$ confluency. After the desired confluency was achieved, serum content in the culture medium was deprived by washing the cells with Phosphate Buffered Saline (PBS, Lonza, Basel, Switzerland) prior to medium changing into serum-free medium (MEM Alpha) without phenol red. The cells were then cultured under following conditions: normoxia $\left(21 \% \mathrm{O}^{2}\right)$ or hypoxia $(1,5$ and $10 \%$ O2) conditions, $n=3$. Secretome collection points were conducted at 48 hours, 72 hours, and 96 hours. To mimic hypoxia condition, the cells were incubated in a tri-gas incubator (Heracell Vios 160i, Thermo Scientific Heraeus, Germany) with humidified gas mixtures of $\mathrm{O}_{2}, \mathrm{CO}_{2}$, and $\mathrm{N}_{2}$. Collected secretome was then aliquoted into an appropriate volume and stored at $-80 \mathrm{oC}$ until further analysis.

\section{Survival and Proliferation of hUC-MSC under Hypoxia}

At the end of the cultivation, the cells were washed with PBS, trypsinized and incubated at $37^{\circ} \mathrm{C}$ in a flask for approximately 7 minutes. The flask was gently shaked to physically detached the cells and collected cells were further centrifuged for 4 minutes at $160 \mathrm{~g}$. The pelleted cells were then resuspended in $1 \mathrm{~mL}$ of basal medium to be further counted using a haemocytometer (Brand GMBH $+\mathrm{CO}$ KG, Wertheim, Germany).

\section{Cell Morphological Changing Observation}

Photomicrographs of hUC-MSCs from all hypoxia groups at all time points were taken from 5 designated locations, from each corner (4 locations) and in the middle (1 location) of the flask. Comparisons of cells from these photomicrographs were performed by 3 different examiners.

\section{Growth Factor Measurement by ELISA}

The collected secretome was analyzed by enzyme-linked immunosorbent assay (ELISA) using vascular endothelial factor (VEGF), neurotrophic growth factor (NGF), and brain-derived neurotrophic factor (BDNF) Immunoassays (R\&D Systems) according to the manufacturers' protocol. All measurements were done in 3 replications together with step-wise dilution standard. The absorbance was read using microplate reader (BioRad, California, USA) at $450 \mathrm{~nm}$ with the correction at $540 \mathrm{~nm}$. ELISA results for neurotrophic growth factors were analyzed in 4 parameter logistics regression tests using software analyzer (MyAssays).

\section{Statistical Analysis}

The data are presented as mean \pm standard deviation. After all of the data passed the normality test, statistical comparisons were performed using one-way analysis of variance (ANOVA) as appropriate according to data distribution $(n=3)$. Significant differences were further investigated using Tukey's HSD multiple comparison test. The significance level was set at $p<0.05$.

\section{Results}

\section{Isolated hUC-MSCs Represented MSCs Characteristics} The isolated cells showed MSCs positive markers as follows: CD73 (99.26\%), CD90 (99.52\%), CD105 (99.47\%), and were lack of CD14 (0.00\%) and CD45 (0.26\%) (Figure 1). The isolated cells highly expressed positive MSCs surface markers, therefore indicating that those cells were MSCs as defined by ISCT.

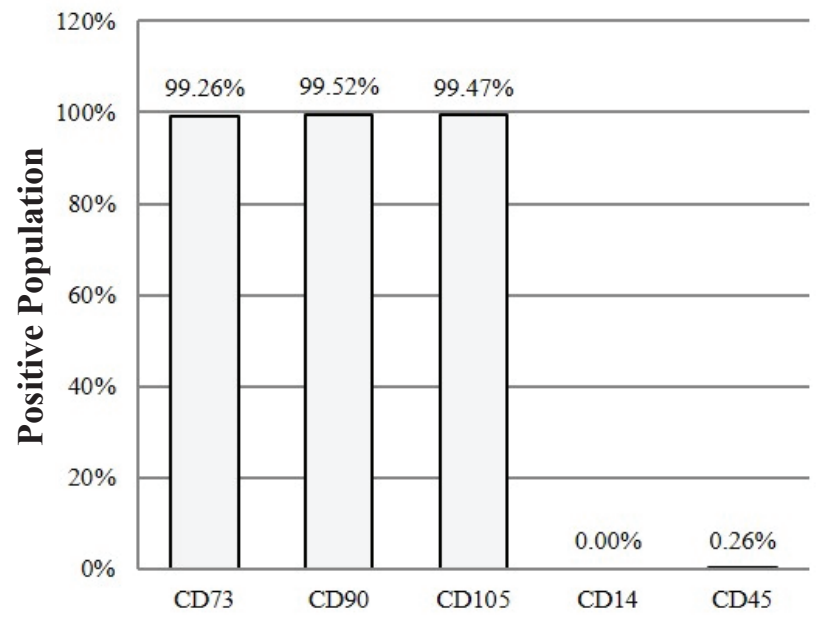

Figure 1. Characterization of hUC-MSCs. The phenotypic markers CD73, CD90, CD105, CD14, dan CD45 were assessed by flow cytometry. Positive populations of surface markers CD73, CD90, and CD105, also the lack of surface markers CD14 and CD45, confirmed that these cells are MSCs.

\section{hUC-MSCs Proliferation was Found Higher in Hypoxia} Compared to Normoxia

The hUC-MSCs showed an increase of cell number from the basal level ( 0 hour) after 48 hours in normoxia or hypoxia conditions with different proliferation rates (Figure 2a). Cells in normoxia condition showed 2.86-fold increase of proliferation rate, while cells in $10 \%, 5 \%$ and $1 \%$ hypoxia 
a

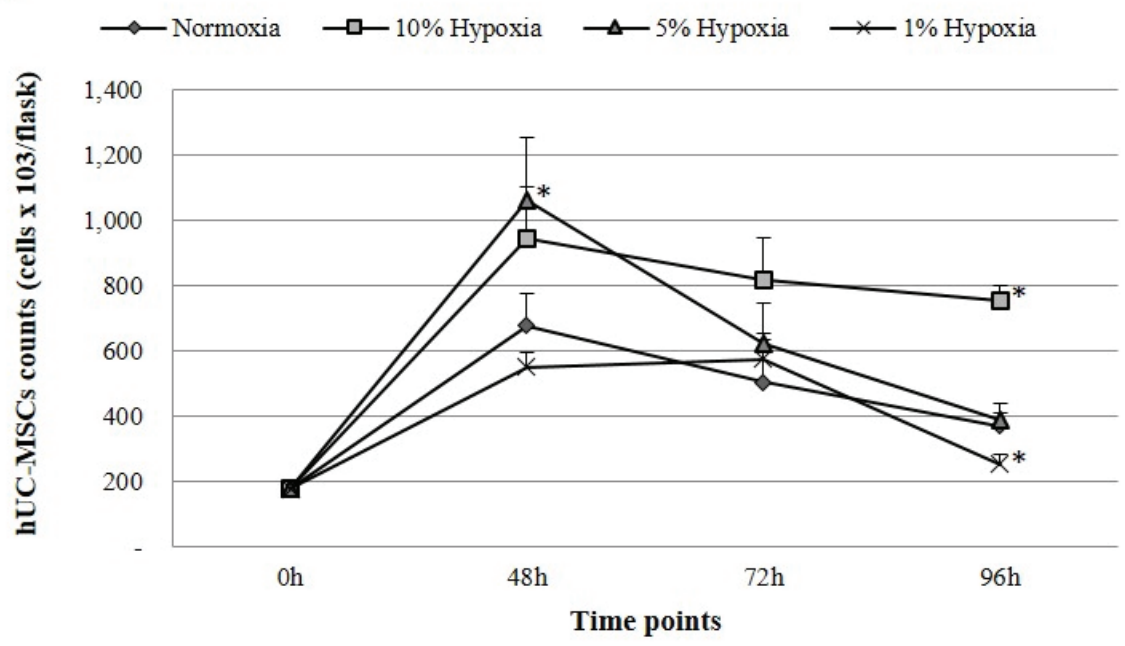

b

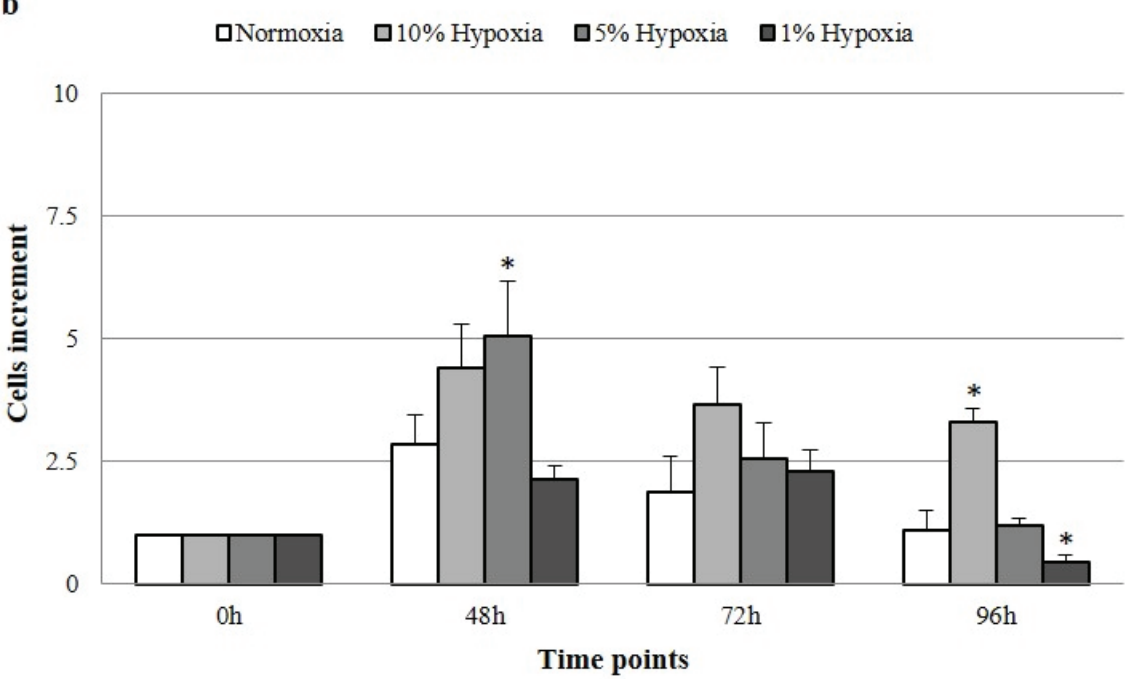

Figure 2. hUC-MSCs counts (a) and cells increment (b) from normoxic/hypoxic conditions at 3 time points. Significant difference with normoxia is represented with * $(p<0.05)$.

showed increases of 4.4 fold, 5.06 fold and 2.14 fold, respectively, of proliferation rate (Figure $2 b$ ).

After 72 hours incubation, almost all cell groups showed a reduction in cell number compared to 48 hours incubation at a range of $16.6 \%-49.4 \%$, except for cells in $1 \%$ hypoxia condition that showed slight increment of cell number (14\%). The reduction of cell number was higher at 96 hours incubation than that at 48 hours incubation in all cell groups at a range of $24 \%-76.3 \%$. The most optimum proliferation rate was found at 48 hours incubation in 5\% hypoxia condition (Figure 2a, 2b). This was then followed by the reduction of cell number in longer incubations.

\section{Hypoxia did Not Gave Significantly Different Morphology to hUC-MSCs}

The morphology of cells cultured under normoxia conditions showed no significant difference on each time points (Figure 3a-3c). After the exposure of 10\% and 5\% hypoxia conditions for 48 hours and 72 hours, all cells showed reduction in size and became slimmer in appearance, especially at 96 hours (Figure 3d-3i).

Cells cultured under $1 \%$ hypoxia conditions at 72 hours and 96 hours showed tendency to form clump (Figure $3 \mathrm{j}-31$, circled) and more of dead cells were found.

\section{Hypoxia Induced Significant VEGF Secretion, Mild BDNF Secretion and No NGF Secretion.}

The neurotrophic factors NGF, BDNF, and VEGF released by hUC-MSCs under normoxic and hypoxic conditions were quantified by ELISA. There were no detectable NGF secretion at any time point cultured in both normoxia and hypoxia.

In normoxia group, the BDNF secretion was induced at 48 hours time point and reached the highest level at an 


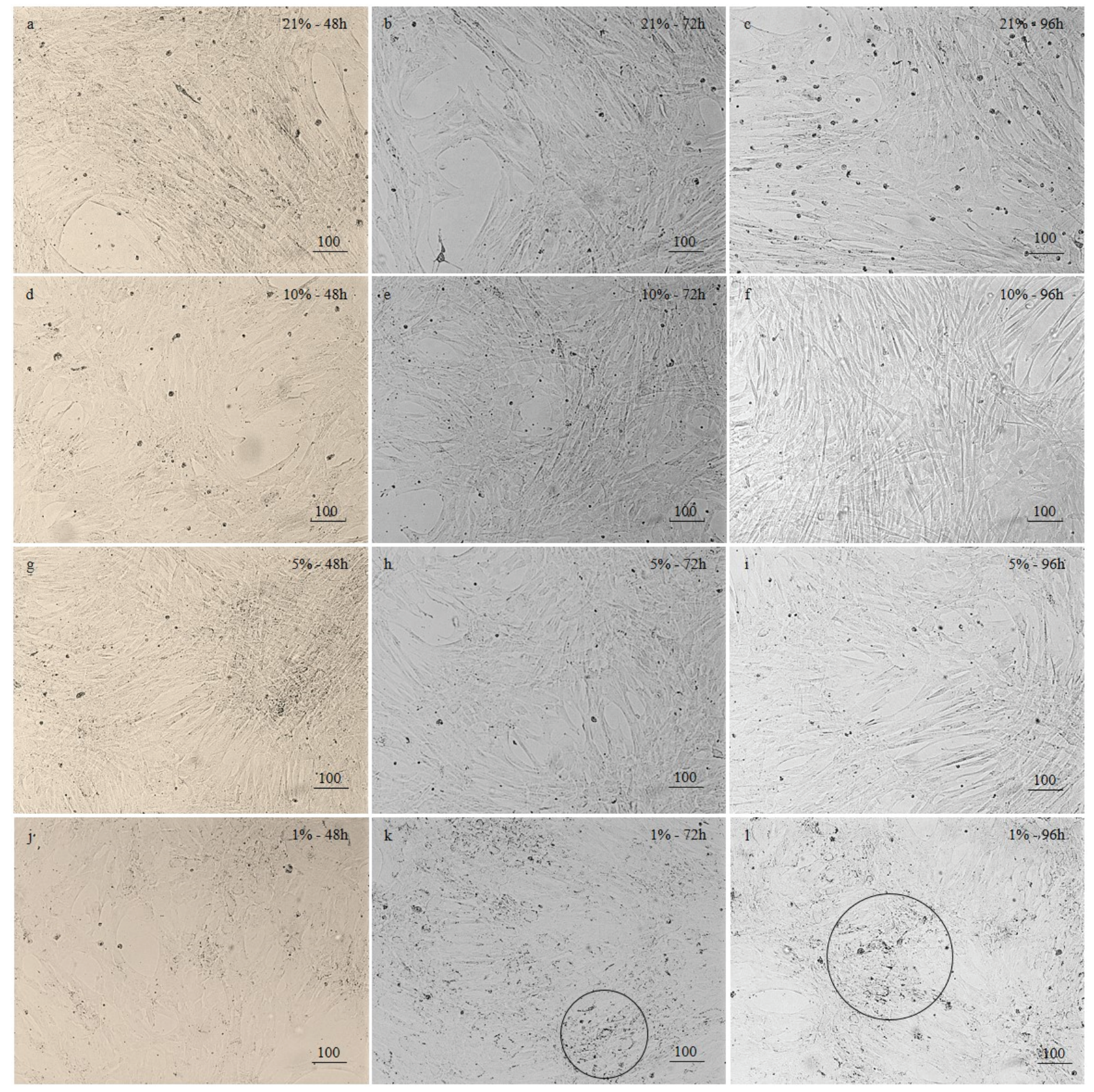

Figure 3. Photomicrograph of hUC-MSCs from normoxic/hypoxic conditions and 3 time points. a. Normoxic, 48 h; b. Normoxic, 72 h; c. Normoxic, 96 h; d. 10\% hypoxic, 48 h; e. 10\% hypoxic, 72 h; f. 10\% hypoxic, 96 h; g. 5\% hypoxic, 48 h; h. $5 \%$ hypoxic, 72 h; i. $5 \%$ hypoxic, $96 \mathrm{~h} ; \mathrm{j} .1 \%$ hypoxic, $48 \mathrm{~h}$; k. 1\% hypoxic, $72 \mathrm{~h} ; 1.1 \%$ hypoxic, $96 \mathrm{~h}$. No particular issues or differences regarding cell morphology between hUC-MSCs cultured in normoxic and hypoxic conditions, in any culture durations. However, hUC-MSCs cultured under $1 \%$ hypoxic grew bigger in size with the tendency to form clump (circled). bar: $100 \mu \mathrm{m}$.

average of $181.9 \pm 13.01 \mathrm{pg} / \mathrm{mL}$ at 96 hours (Figure 4a). This secretion increment was 1.7 fold higher compared to 48 hours time point. The hypoxia exposure from 1-10\% oxygen levels induced the BDNF secretion at a lower level compared to the normoxia group at the studied time points. This finding indicated that normoxia had better ability to induce BDNF compared to hypoxia.
In contrast to the BDNF secretion, VEGF secretion was barely detectable in normoxia. However, in 5\% hypoxia at 96 hours, the VEGF secretion reached the highest level at an average of $7707.55 \pm 2110.85 \mathrm{pg} / \mathrm{mL}(p<0.05$; Fig. 4b). This result indicated that VEGF was very sensitive to oxygen tension changes and long-time exposure of low oxygen level, especially at $5 \%$ hypoxia. 
a

\section{BDNF}
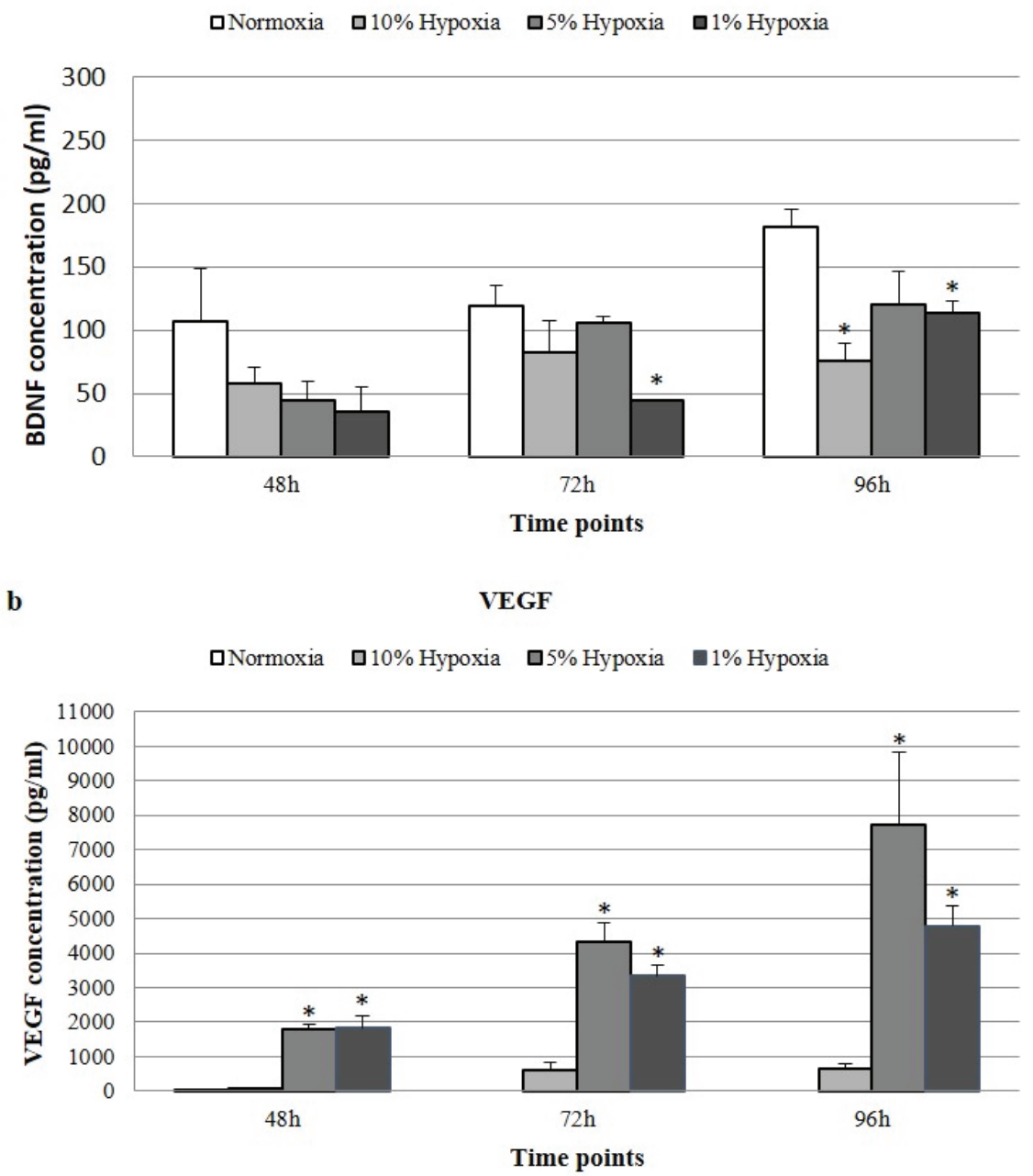

Figure 4. Comparison of neurotrophic growth factors measured in the medium under normoxic/hypoxic conditions and collected after 3 time points. The growth factors were analyzed by ELISA for NGF (not shown), BDNF, and VEGF. Significant difference with normoxia is represented with $*(\mathrm{p}<0.05)$.

Taken together, when we compared BDNF concentration to VEGF concentration in a ratio (Table 1), a gradual changing along with culture duration was found. The ratio was gradually increasing in 5\% and $10 \%$ hypoxia, however the ratio in $1 \%$ hypoxia showed decreased value in longer culture duration. These results suggested that in hypoxic condition, the increment in VEGF concentration was much higher than BDNF concentration that could change BDNF to VEGF ratio dramatically.

\section{Discussion}

In this study, we have explored the potency of hUC-MSCs to release neurotrophic growth factors in amino acid-rich medium and under various hypoxic conditions. We chose commercial medium like MEM Alpha that contained higher concentration of non-essential amino acid such as alanine, aspartic acid, asparagine, and glutamic acid, also higher concentration of conditionally non-essential amino acid such as glycine, cysteine and proline (Supplemental Data) compared to DMEM F12 that had been commonly used in previous studies.(14,19-21) FBS was avoided in these experiments since the presence of cytokines contained in FBS would complicate the analysis.

A study by Majumdar, et al., reported that human umbilical cord WJ-MSCs which were cultured in DMEM F12 under 2\% hypoxia condition showed a gradual decrease of cell number from 24 hours until 96 hours incubation with the lowest cell percentage at $24 \%$ in 96 hours incubation. (14) This result was different from ours that found an increase of cell number in hypoxia condition at 48 hours incubation followed was slightly different from ours that found a constant number of harvested cells when incubated 
Table 1. The BDNF:VEGF ratio at 3 time points and various oxygen tensions.

\begin{tabular}{ccc}
\hline Time Points & Oxygen Tension & $\begin{array}{c}\text { BDNF : VEGF } \\
\text { Ratio }\end{array}$ \\
\hline \multirow{3}{*}{48 hours } & $21 \%$ & $1: 0.03$ \\
& $10 \%$ & $1: 1.21$ \\
& $5 \%$ & $1: 40.17$ \\
72 hours & $1 \%$ & $1: 51.24$ \\
\hline \multirow{7}{*}{96 hours } & $21 \%$ & $1: 0$ \\
& $10 \%$ & $1: 7.23$ \\
& $5 \%$ & $1: 41.11$ \\
& $1 \%$ & $1: 74.64$ \\
\hline & $10 \%$ & $1: 0$ \\
& $1 \%$ & $1: 8.38$ \\
\hline
\end{tabular}

for 48 hours and 72 hours but decrease significantly in 96 hours time point. Similar to Majumdar's study, we might conclude that prolong incubation for 96 hours resulted in apoptosis fate.(14) The $1 \%$ hypoxic condition that we used in this experiment could be a lethal condition for the cells since it was not the MSCs niche because MSCs need physiologic $\mathrm{O}_{2}$ level which was approximately at $2 \%-8 \%$ $\mathrm{O}_{2}$ level as described by Mohyeldin, et al., to be able to give molecular and cellular responses.(22)

Drela, et al., found that the morphology of the WJMSCs cultured in human MSC growth medium under 5\% hypoxia for 2-4 weeks is relatively smaller in size and round, with a tendency to lay one on the top of another at the center of proliferation and forming colonies (colony-forming unit fibroblasts).(23) In contrast, the cells grew under normoxia rarely formed similar centers of proliferation. These findings are similar with ours, considering that the culture time in our study were only up to 96 hours, but we only found clumped cells under $1 \%$ hypoxia at 72 hours and 96 hours time points.

In our study, BDNF level had higher secretion under normoxia than hypoxia, this is similar to previous study.(14) This result suggests that the BDNF expression and secretion does not rely on the oxygen tension level and a normal oxygen level gives rise to the optimum BDNF secretion. Under considerably the same conditions with other study (14), despite higher release of VEGF, the BDNF release in our study was lower. This suggest that BDNF might need different composition of nutrients in the culture medium.

In contrast, NGF level did not have any changes at all, both in normoxia and hypoxia at any time points. This result suggested that NGF was not secreted by MSCs despite oxygen tension changing. We assume that NGF release is tissue-related.

We found that hypoxia condition, especially at 5\% hypoxia, significantly increased VEGF secretion with the highest at 72 hours time point. This finding is similar with previous report (24), but different with other (14) that the release of VEGF was significantly higher under hypoxia conditions up until 48 hours time point then decreased considerably by 72 hours of incubation. On the contrary, another study (12) barely detected VEGF in secretome of human umbilical cord perivascular cells (hUC-PVCs) cultured under normoxia (except at 72 hours). This difference might be related to the different composition of the culture medium or source of cell used in our studies.

VEGF release in our study was significantly higher under hypoxia condition than normoxia. This might be due to the upregulation of hypoxia inducible factor (HIF)1 alpha in hypoxia which will increase the expression of VEGF.(25) The release of VEGF was also higher compared with other study under relatively same conditions in our study (14), at approximately 7 fold concentration of their study. Such high release of VEGF was a surprising result since the hUC-MSC was under stress condition because of the limited oxygen tension which at a certain point might blunt the cells' ability to produce growth factors and to survive. We assume this might be related to the different composition of culture medium used.

We assume that despite of hypoxic mechanism that induced VEGF secretion, there are other additional mechanisms that work synergistically with the hypoxic mechanism to give high increasing fold of VEGF release. Since the concentrations of several amino acids content in our study were higher than previous studies, we are considering that amino acids might play an important role as additional mechanisms to induce VEGF secretion.

Hypoxia condition is known to induce VEGF secretion via HIF-1 alpha signaling pathway (25) and a recent study by Lee, et al., revealed that there is a novel mechanism of cell proliferation and migration via HIF-1 alpha/fatty acid synthase (FASN) axis, which activates the mammalian target of rapamycin complex 1 (mTORC1) (26).

mTORC1 is a complex composed of the protein kinase mTOR, regulator-associated protein of mTOR (Raptor), mammalian lethal with SEC13 protein 8 (mLST8; G $\beta$ L), proline-rich Akt substrate $40 \mathrm{kDa}$ (PRAS40), and DEP domain containing mTOR-interacting protein (Deptor), Tti1, and Tel2 $(27,28)$. This mTORC1 complex is activated by several metabolic resources such as amino acids, energy 
sources, growth factors, cytokines, and hormones and hypoxia condition. $(26,27)$

Amino acids take part in mTORC1 activation by priming and activating, which are two discrete and separable steps.(29) The group of priming amino acids includes L-asparagine, L-glutamine, L-threonine, L-arginine, L-glycine, L-proline, L-serine, L-alanine, and L-glutamic acid. The group of activating amino acids includes L-leucine, L-methionine, L-isoleucine, and L-valine. MEM alpha contains higher concentration of L-asparagine, L-glycine, L-proline, L-alanine and L-glutamic acid that all belong to priming amino acids for mTORC1 activation. Although activating amino acids contents in MEM alpha are present in moderate concentrations however such amounts might be functional to activate mTORC1.

The limitations of our study are we did not explore the gene regulation mechanisms in this study, so we do not know the exact mechanisms of VEGF induction in our study, thus further investigation is needed to clarify the exact mechanism of high secretion of VEGF found in our study.

Furthermore, VEGFs are a family of secreted polypeptides with a highly conserved receptor-binding cystine-knot structure similar to platelet-derived growth factors.(30) There are five members of VEGF family in mammals: VEGF-A, placenta growth factor (PGF), VEGF-B, VEGF-C, and VEGF-D. VEGF-A is the most abundant and the prototypical member of this family. Studies on VEGF-A have given most of the information about localization and expression of all of the VEGFs.(30) Hypoxia upregulates VEGF-A gene expression $(25,30)$, but not VEGF-B and VEGF-C (31). About VEGF-D, little is known whether hypoxia or other factors upregulate it (31) but some reports found that VEGF-D expression is upregulated in tumors $(32,33)$. We did not measure each of the VEGF family members secreted by hUC-MSCs since our ELISA kit was only able to measure total VEGF. Further investigation using a more specific ELISA kit for each VEGF family members secreted by hUC-MSCs can give more information about VEGF family members most affected by exposure in our study.

\section{Conclusion}

In conclusion, a combination of amino acid-rich medium and hypoxia condition induced dramatically high VEGF secretion by hUC-MSCs. However, such combination did not have any significant effect toward the secretion of NGF and BDNF.

\section{Acknowledgement}

We want to thank the Stem Cell and Cancer Institute, PT. Kalbe Farma, Tbk. for giving us the stock cells and providing the laboratory facility for our study. The authors have declared that no competing interests exist.

The research was funded by the Ministry of Research, Technology and Higher Education, Indonesia (No. 1072/III/ LPPM-HD/05/2017).

\section{References}

1. Friedenstein AJ, Piatetzky-Shapiro II, Petrakova KV. Osteogenesis in transplants of bone marrow cells. J Embryol Exp Morphol. 1966; 16: 381-90.

2. Meirelles L da S, Chagastelles PC, Nardi NB. Mesenchymal stem cells reside in virtually all post-natal organs and tissues. J Cell Sci. 2006; 119: 2204-13.

3. Wang HS, Hung SC, Peng ST, Huang CC, Wei HM, Guo YJ, et al. Mesenchymal stem cells in the Wharton's jelly of the human umbilical cord. Stem Cells. 2004; 22: 1330-7.

4. Dominici M, Le Blanc K, Mueller I, Slaper-Cortenbach I, Marini FC, Krause DS, et al. Minimal criteria for defining multipotent mesenchymal stromal cells. The International Society for Cellular Therapy position statement. Cytotherapy. 2006; 8: 315-7.

5. Lindvall O, Kokaia Z. Stem cells in human neurodegenerative disorders - time for clinical translation? J Clin Invest. 2010; 120: 29-40.

6. Gögel S, Gubernator M, Minger SL. Progress and prospects: Stem cells and neurological diseases. Gene Ther. 2011; 18: 1-6. doi: 10.1038/gt.2010.130.

7. Xu X, Yi F, Pan H, Duan S, Ding Z, Yuan G, et al. Progress and prospects in stem cell therapy. Acta Pharmacol Sin. Nature Publishing Group. 2013; 34: 741-6.

8. Dalous J, Larghero J, Baud O. Transplantation of umbilical cordderived mesenchymal stem cells as a novel strategy to protect the central nervous system: technical aspects, preclinical studies, and clinical perspectives. Pediatr Res. 2012; 71(4 Pt 2): 482-90.

9. Lavoie JR, Rosu-Myles M. Uncovering the secretes of mesenchymal stem cells. Biochimie. Elsevier Masson SAS; 2013; 95: 2212-21.

10. Baglio SR, Pegtel DM, Baldini N. Mesenchymal stem cell secreted vesicles provide novel opportunities in (stem) cell-free therapy. Front Physiol. 2012; 6: 359. doi: 10.3389/fphys.2012.00359.

11. Lee SC, Kim KH, Kim OH, Lee SK, Hong HE, Won SS, et al. Determination of optimized oxygen partial pressure to maximize the liver regenerative potential of the secretome obtained from adipose-derived stem cells. Stem Cell Res Ther. 2017; 8: 181. doi: 10.1186/s13287-017-0635-x.

12. Ribeiro CA, Fraga JS, Grãos M, Neves NM, Reis RL, Gimble JM, et al. The secretome of stem cells isolated from the adipose tissue and Wharton jelly acts differently on central nervous system derived cell populations. Stem Cell Res Ther. 2012; 3: 18. doi: 10.1186/ scrt109.

13. Hsieh JY, Wang HW, Chang SJ, Liao KH, Lee IH, Lin WS, et al. Mesenchymal stem cells from human umbilical cord express preferentially secreted factors related to neuroprotection, neurogenesis, and angiogenesis. PLoS One. 2013; 8: e72604. doi: 10.1371/journal.pone.0072604. 
14. Majumdar D, Bhonde R, Datta I. Influence of ischemic microenvironment on human Wharton's jelly mesenchymal stromal cells. Placenta. 2013; 34: 642-9.

15. Stolzing A, Coleman N, Scutt A. Glucose-induced replicative senescence in mesenchymal stem cells. Rejuvenation Res. 2006; 9: $31-5$.

16. Saki N, Jalalifar MA, Soleimani M, Hajizamani S, Rahim F. Adverse effect of high glucose concentration on stem cell therapy. Int $\mathrm{J}$ Hematol Stem Cell Res. 2013; 7: 33-9.

17. Hagmann S, Moradi B, Frank S, Dreher T, Kämmerer PW, Richter W, et al. Different culture media affect growth characteristics, surface marker distribution and chondrogenic differentiation of human bone marrow-derived mesenchymal stromal cells. BMC Musculoskelet Disord. 2013;14: 223. doi: 10.1186/1471-2474-14-223.

18. Nekanti U, Rao VB, Bahirvani AG, Jan M, Totey S, Ta M. Long-term expansion and pluripotent marker array analysis of Wharton's jellyderived mesenchymal stem cells. Stem Cells Dev. 2010; 19: 117-30.

19. Govindasamy V, Ronald VS, Totey S, Binti Din S, Mustafa WMBW, Totey S, et al. Micromanipulation of culture niche permits long-term expansion of dental pulp stem cells-an economic and commercial angle. Vitr Cell Dev Biol - Anim. 2010; 46: 764-73.

20. Zhao Q, Hu J, Xiang J, Gu Y, Jin P, Hua F, et al. Intranasal administration of human umbilical cord mesenchymal stem cellsconditioned medium enhances vascular remodeling after stroke. Brain Res. 2015; 1624: 489-96.

21. Zhu SF, Zhong ZN, Fu XF, Peng DX, Lu GH, Li WH, et al. Comparison of cell proliferation, apoptosis, cellular morphology and ultrastructure between human umbilical cord and placentaderived mesenchymal stem cells. Neurosci Lett. 2013; 541: 77-82.

22. Mohyeldin A, Garzón-Muvdi T, Quiñones-Hinojosa A. Oxygen in stem cell biology: A critical component of the stem cell niche. Cell Stem Cell. 2010; 7: 150-61.

23. Drela K, Sarnowska A, Siedlecka P, Szablowska-Gadomska I, Wielgos M, Jurga M, et al. Low oxygen atmosphere facilitates proliferation and maintains undifferentiated state of umbilical cord mesenchymal stem cells in an hypoxia inducible factor-dependent manner. Cytotherapy. 2014; 16: 881-92.

24. Liu L, Gao J, Yuan Y, Chang Q, Liao Y, Lu F. Hypoxia preconditioned human adipose derived mesenchymal stem cells enhance angiogenic potential via secretion of increased VEGF and bFGF. Cell Biol Int. 2013; 37: 551-60.

25. Forsythe JOA, Jiang B, Iyer NV., Agani F, Leung SW. Activation of vascular endothelial growth factor gene transcription by hypoxiainducible factor 1. Mol Cell Biol. 1996; 16: 4604-12.

26. Lee HJ, Ryu JM, Jung YH, Oh SY, Lee SJ, Han HJ. Novel pathway for hypoxia-induced proliferation and migration in human mesenchymal stem cells: Involvement of HIF-1 $\alpha$, FASN, and mTORC1. Stem Cells. 2015; 33: 2182-95.

27. Kim SG, Buel GR, Blenis J. Nutrient regulation of the mTOR complex 1 signaling pathway. Mol Cells. 2013; 35: 463-73.

28. Zheng X, Liang Y, He Q, Yao R, Bao W, Bao L, et al. Current models of mammalian target of rapamycin complex 1 (mTORC1) activation by growth factors and amino acids. Int J Mol Sci. 2014; 15: 20753 69.

29. Dyachok J, Earnest S, Iturraran EN, Cobb MH, Ross EM. Amino acids regulate mTORC1 by an obligate two-step mechanism. J Biol Chem. 2016; 291: 22414-26.

30. Holmes DIR, Zachary I. The vascular endothelial growth factor (VEGF) family: angiogenic factors in health and disease. Genome Biol. 2005; 6: 209. doi: 10.1186/gb-2005-6-2-209.

31. Li X, Eriksson U. Novel VEGF family members: VEGF-B, VEGF-C and VEGF-D. Int J Biochem Cell Biol. 2001; 33: 421-6.

32. White JD, Hewett PW, Kosuge D, McCulloch T, Enholm BC, Carmichael J, et al. Vascular endothelial growth factor-D expression is an independent prognostic marker for survival in colorectal carcinoma. Cancer Res. 2002; 62: 1669-75.

33. Yasuoka H, Nakamura Y, Zuo H, Tang W, Takamura Y, Miyauchi A, et al. VEGF-D expression and lymph vessels play an important role for lymph node metastasis in papillary thyroid carcinoma. Mod Pathol. 2005; 18: 1127-33. 